[Short Note]

\title{
Design of Hardware-Based Biomimetic Neural Networks Exhibiting Oscillatory Burst Firing of Lamprey Nervous System
}

\author{
Katsuyuki Morishita*, Yuki Takei*, and Ken Saito** \\ *Precision Machinery Eng., Graduate School of Science and Technology, Nihon University, Chiyoda-ku, Tokyo 101-8308, Japan \\ **Department of Precision Machinery Eng., College of Science and Technology, Nihon University, Funabashi-shi, Chiba 274-8501, Japan
}

(Received March 19, 2020; accepted June 10, 2020, published July 29, 2020)

\begin{abstract}
We designed hardware-based neural networks (HNNs) that resemble neural functions of the lamprey using electronic circuits. The lamprey has a relatively simple nervous system among vertebrates. In addition, the central pattern generator of the lamprey is better understood than that of other vertebrates. Hence, we designed the HNNs based on the known structure of the lamprey nervous system. The HNNs can generate burst firing that imitates two functions of lamprey. In one function, the left and right segments alternately generate oscillatory burst firing. In the other function, burst firing propagates from head to tail of the lamprey. Therefore, we achieved biomimetic HNNs that generate oscillatory activity from the lamprey nervous system.
\end{abstract}

Keywords: Hardware-Based Neural Networks, Robot, Lamprey, Biomimetics, Burst Firing

\section{Introduction}

In general, animals can move autonomously in their environments given their excellent motor control. In contrast, robot systems mostly controlled by computer programs cannot handle all unknown situations. Consequently, a human operator must control a robot system under complicated scenarios.

Mimicking animal behavior may allow to enhance robot control. Some studies on the somatic nervous system have been focused on the central pattern generator (CPG). $[1,2]$ The CPG comprises neural networks in the spinal cord and is thought to control movement patterns such as walking and swimming. As the nervous system of the lamprey is better understood than that of other vertebrates by its simplicity, $[3,4]$ its functions may be implemented in robotic systems.

Software or hardware-based models can be used to imitate the nervous system. Software models are more widely used because they are easy to design. For example, the AmphiBot robot uses software models[5] to implement a biomimetic CPG of the lamprey nervous system for movement control, achieving movements resembling those of a lamprey. In small robots, hardware-based models can be implemented more easily than software models. However, hardware-based models are more costly and time consuming to design than software models. Therefore, few studies have addressed hardware-based models.

Although biomimetic hardware-based neural models of the nervous systems of different organisms have been studied, [6, 7] conventional networks have failed to realistically imitate their structure and function. In this study, we designed hardware-based neural networks (HNNs) that replicate the structure and functions of the lamprey CPGs. The designed HNNs generate periodic burst waveforms that imitate two lamprey functions. Simulation results confirm the output waveforms generated by the developed HNNs.

\section{Biomimetic HNNs Imitating Lamprey Nervous System}

A. HNN components

Figure 1 shows the circuit diagrams of a cell body model and a synapse model that constitute the basic HNN components. The cell body model is an oscillator circuit that

This work was supported by JSPS KAKENHI Grant Number JP18K04060 and supported by Research Institute of Science and Technology, Nihon University College of Science and Technology, Leading Research Promotion Grant. 


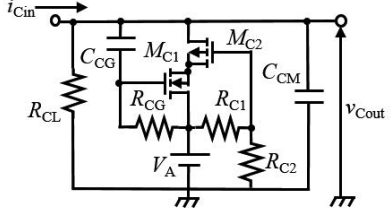

(a)

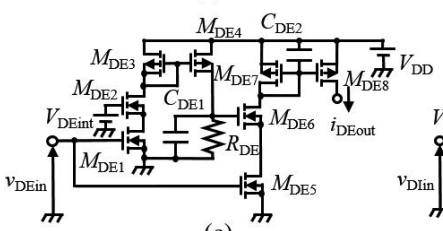

(c)

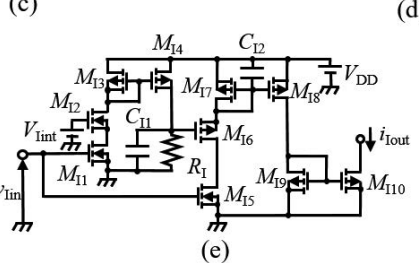

Fig. 1. Circuit diagrams of cell body and synapse models.

imitates some neural functions (Fig. 1(a)) and can switch between the self-excitation mode and separately excited mode by changing the voltage of source $V_{\mathrm{A}}$. The synaptic model is a circuit that performs the spatiotemporal sum of the outputs from the cell body model. Figures 1(b) and (c) show excitatory synaptic models that promote the oscillation of the corresponding output of a cell body model when the cell body model of the input oscillates. Figures $1(d)$ and (e) show inhibitory synaptic models that inhibit the oscillation of the corresponding output of a cell body model when the cell body model of the input oscillates. In addition, the HNNs include synaptic models that provide delay using integrator circuits (Figs. 1(c) and (d)), and synaptic models whose output decreases after a predefined time (Fig. 1(e))

The voltage source for the self-excited oscillation is $V_{\mathrm{A}}$, and that for the separately excited oscillation is $V_{\mathrm{As}}$. The circuit parameters are the following. Resistors: $R_{\mathrm{CL}}=3.3$ $\mathrm{k} \Omega, R_{\mathrm{CG}}=820 \mathrm{k} \Omega, R_{\mathrm{C} 1}=18 \mathrm{k} \Omega, R_{\mathrm{C} 2}=20 \mathrm{k} \Omega, R_{\mathrm{DE}}=39 \mathrm{k} \Omega$, $R_{\mathrm{DI}}=39 \mathrm{k} \Omega$, and $R_{\mathrm{I}}=47 \mathrm{k} \Omega$. Capacitors: $C_{\mathrm{CG}}=1 \mathrm{nF}$, $C_{\mathrm{CM}}=12 \mathrm{nF}, C_{\mathrm{E}}=100 \mathrm{nF}, C_{\mathrm{DE} 1}=3.3 \mu \mathrm{F}, C_{\mathrm{DE} 2}=1 \mathrm{pF}$, $C_{\mathrm{DI} 1}=C_{\mathrm{DI} 2}=C_{\mathrm{I} 1}=10 \mu \mathrm{F}$, and $C_{\mathrm{I} 2}=20 \mu \mathrm{F}$. Voltage sources: $V_{\mathrm{A}}=3.5 \mathrm{~V}, V_{\mathrm{AS}}=3.45 \mathrm{~V}, V_{\mathrm{DD}}=3.5 \mathrm{~V}, V_{\text {Eint }}=1.5 \mathrm{~V}, V_{\text {DEint }}=$ $1.6 \mathrm{~V}, V_{\text {Dlint }}=1.65 \mathrm{~V}, V_{\text {lint }}=1.62 \mathrm{~V}$. In addition, we used n-type MOSFETs (metal-oxide-semiconductor field-effect transistors) model SSM3K17FU and p-type MOSFETs model BSS83P for $M_{\mathrm{I} 5}$ and model BSH203 for the other transistors.

\section{B. HNN structures}

Figure 2 shows the connection diagram of the HNNs to replicate the lamprey nervous system. We devised the

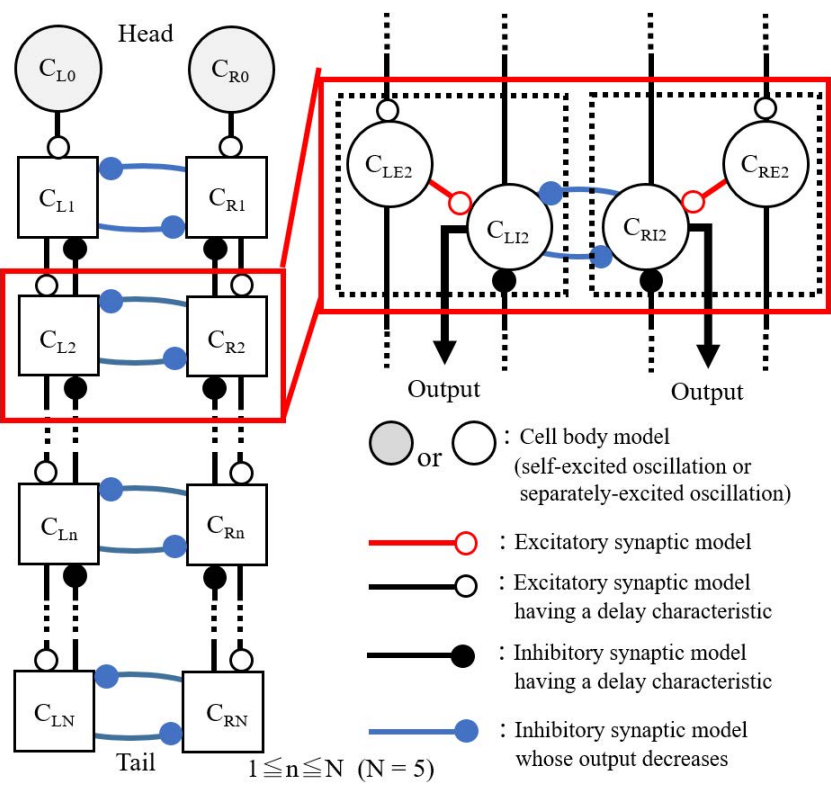

Fig. 2. Connection diagram of HNNs.

model structure by simplifying the lamprey CPG structure. The HNNs are bilaterally symmetric like the lamprey CPG. The inhibitory synaptic models reciprocally inhibit each other in opposite cell body models. The output of the mutually inhibiting synaptic models decreases after some time.

With the designed structure, the HNNs imitate the function of lateral inhibitory interneurons. In the reciprocally inhibitory cell body models, when one model oscillates, the other cannot oscillate. In addition, the models alternately exhibit oscillatory bursts. The cell body models are connected from head to tail by excitatory synaptic models that provide delay characteristics. Therefore, an oscillation of the cell body models $\left(\mathrm{C}_{\mathrm{L} 0}, \mathrm{C}_{\mathrm{R} 0}\right)$ in the self-excitation mode propagates to the input of the other cell body models with a delay. Moreover, the cell body models are connected from tail to head by inhibitory synaptic models with delay to stabilize the lag period.

\section{Simulation results}

Figure 3 shows an example of the output waveforms from the HNNs. The output voltage of each cell body model, $v_{\text {Cout }}$, is shown in Fig. 1(a). These simulation results were obtained using PSpice (Cadence Design Systems). Figure 3 (a) shows the output waveforms from $\mathrm{C}_{\mathrm{L} 1}$ and $\mathrm{C}_{\mathrm{R} 1}$. Figure $3(\mathrm{~b})$ shows the output waveforms from $\mathrm{C}_{\mathrm{L} 1}-\mathrm{C}_{\mathrm{L} 4}$. In Fig. 3(a), $C_{L 1}$ and $C_{R 1}$ alternately generate oscillatory bursts. The burst oscillation resembles the neural function of the lamprey in which the left and right segments alternately oscillate. In Fig. 3(b), the oscillatory bursts propagates with delays from $\mathrm{C}_{\mathrm{L} 1}$ to $\mathrm{C}_{\mathrm{L} 4}$. These bursts resemble the neural function in which burst the oscillation propa- 


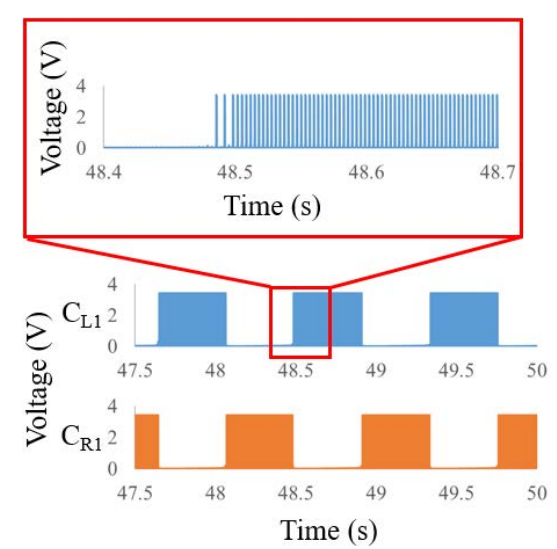

(a)

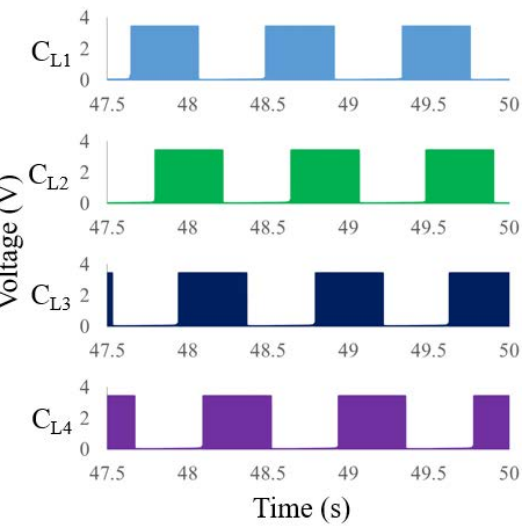

(b)

Fig. 3. HNN output waveforms.

gates from head to tail of the lamprey. Therefore, the HNNs generate biomimetic oscillatory burst firing of functions from the lamprey $\mathrm{CPG}$.

We plan to use shape-memory alloys as actuators for a lamprey-type robot. This alloy shrinks at high temperatures and expands at low temperatures. The output waveforms shown in Fig. 3 can be used to drive the actuator by heating the alloy during the oscillatory burst period and letting it radiate during the inactivity period. Therefore, by assembling as many actuators as circuits $\mathrm{C}_{\mathrm{LN}}$ and $\mathrm{C}_{\mathrm{RN}}$, we expect the robot to generate snake-like movement.

\section{Conclusion}

We designed HNNs that imitate functions of the lamprey CPG. Specifically, the HNNs generate oscillatory bursts corresponding to two lamprey neural functions. In one function, the left and right segments alternately generate oscillatory bursts, and in the other function, the oscillatory burst propagates from head to tail. We verified the HNN output waveforms by simulations. We are currently constructing the HNNs using discrete circuits, and in future work, we will design a lamprey-type robot equipped with the proposed HNNs for actuation.

\section{Acknowledgment}

We appreciated to the Nihon University Robotics Society (NUROS).

\section{References}

[1] J. R. Cazalets, M. Borde, and F. Clarac, "Localization and organization of the central pattern generator for hindlimb locomotion in newborn rat," Journal of Neu- roscience, Vol. 15, Issue 7, pp. 4943-4951, July 1995.

[2] T. E. Dick, Y. Oku, J. R. Romaniuk, and N. S. Cherniack, "Interaction between central pattern generators for breathing and swallowing in the cat," Journal of Physiology, Vol. 465, Issue 1, pp. 715-730, June 1993.

[3] S. Grillner, T. Deliagina, A. El Manira, R. H. Hill, G. N. Orlovsky, P. Wallén, Ö. Ekeberg, and A. Lansner, "Neural networks that co-ordinate locomotion and body orientation in lamprey," Trends in Neurosciences, Vol. 18, Issue 6, pp. 270-279, 1995.

[4] A. Kozlov, J. H. Kotaleski, E. Aurell, S. Grillner, and A. Lansner, "Modeling of Substance P and 5-HT Induced Synaptic Plasticity in the Lamprey Spinal CPG: Consequences for Network Pattern Generation,” Journal of Computational Neuroscience, Vol. 11, Issue 2, pp. 183-200, September 2001.

[5] A. Crespi, A. J. Ijspeert, and A. Crespi, "Online trajectory generation in an amphibious snake robot using a lamprey-like central pattern generator model,” 2007 IEEE International Conference on Robotics and Automation, Roma, Italy, 10-14 April 2007, pp. 262-268.

[6] K. Saito, M. Ohara, M. Abe, M. Kaneko, and F. Uchikoba, "Gait Generation of Multilegged Robots by using Hardware Artificial Neural Networks," INTECH, 2018, pp. 29-50.

[7] S. Kawamura, D. Tanaka, T. Tanaka, et al., "Neural networks IC controlled multi-legged waiking MEMS robot with independent leg mechanism,” Artificial Life and Robotics, Vol. 23, Issue 3, pp. 380-338, September 2018. 


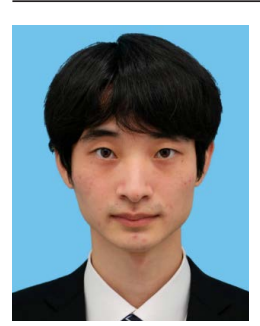

\section{Katsuyuki Morishita}

Katsuyuki Morishita received a B.S. degree from Nihon University in 2019. He is now a master course student of the Precision Machinery Engineering, Graduate School of Science and Technology, Nihon University.

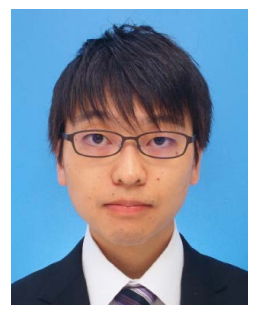

\section{Yuki Takei}

Yuki Takei received a B.S. degree and an M.S. degree from Nihon University in 2017 and 2019. He is now a doctor course student of the Precision Machinery Engineering, Graduate School of Science and Technology, Nihon University.

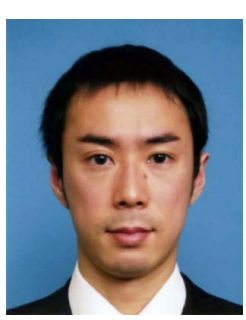

\section{Ken Saito}

Ken Saito received a B.S. degree, an M.S. degree, and a $\mathrm{Ph} . \mathrm{D}$. degree in engineering from Nihon University in 2001, 2004, and 2010, respectively. He was a Research Assistant from 2007 to 2011 and Assistant Professor from 2011 to 2017 in the same university. Also, he was a visiting scholar at the University of California, Berkeley. He is now an Associate Professor at Nihon University. His current research interests include hardware neural networks, neuro-robotics, and microrobot. He is a member of JIEP, IEEE, IEEJ, and so on. 